
\title{
25 Research Square \\ Ciprofloxacin Induced Antibiotic Resistance in Salmonella Typhimurium Mutants and Genome Analysis
}

\section{Aarti Kakatkar}

Bhabha Atomic Research Centre

\section{Anubrata Das}

Bhabha Atomic Research Centre

Ravindranath Shashidhar ( $\nabla$ shashi@barc.gov.in )

Bhabha Atomic Research Centre https://orcid.org/0000-0002-2759-6954

\section{Research Article}

Keywords: Salmonella Typhimurium, ciprofloxacin, WGS, mutation, antibiotic resistance

Posted Date: June 30th, 2021

DOI: https://doi.org/10.21203/rs.3.rs-656905/v1

License: (c) This work is licensed under a Creative Commons Attribution 4.0 International License. Read Full License

Version of Record: A version of this preprint was published at Archives of Microbiology on September 28th, 2021. See the published version at https://doi.org/10.1007/s00203-021-02577-z. 


\section{Abstract}

Salmonella species is an important food-borne pathogen. Ciprofloxacin is used for treatment of salmonellosis. The wild type of Salmonella Typhimurium was exposed to 10 -fold of minimal inhibitory concentration (MIC) of ciprofloxacin. The survivors of the first exposure were regrown. This regrown population was exposed to similar ciprofloxacin treatment. After 20 repetitions of the antibiotic exposure, the survival population was evaluated for antibiotic resistance. The mutants had 100 -fold more of the initial MIC. The mutants were of smaller size $(0.7 \pm 0.5 \mathrm{~mm})$ with a lag phase of $70 \mathrm{~min}$. These mutants were resistant to other antibiotics belonging to different classes but were sensitive to heat and osmotic stress. The whole genome sequencing (WGS) analysis of antibiotic resistant mutants revealed interesting mutation pattern. Total 40513 mutations were observed across the genome (total 3843 annotated genes). Relatively small percentage (5.2\%) of mutations were non-synonymous. Four-fold more transitions were observed than transversions. Ratio of $<1$ transition vs transversion shows a positive selection for antibiotic resistant trait. Seven genes with over 50 mutations were observed. However, mutation distribution across the genome was relatively uniform. The important genes like dnaE, gyrA, iro $C$, metH and $r p o B$ involved in antibiotic resistance had point mutations. The genome analysis showed that most affected pathways were transcription regulation, transmembrane transport, cell adhesion, pathogenesis, pilus assembly, oxidation-reduction mechanisms, phosphor-relay signal transduction and LPS biosynthesis. We propose that development resistance to ciprofloxacin is because of the mutations in these genes.

\section{Introduction}

Salmonella Typhimurium causes approximately 1.35 million illnesses, 26,500 hospitalizations, and 420 deaths each year in the United States (CDC, 2019). A common first line of treatment is fluoroquinolones for elders and azithromycin in infants. However due to increased resistance to fluoroquinolones especially ciprofloxacin (3\%) in US, $56 \%$ in Mexico and $>91 \%$ in Thailand, ceftriaxone has become a drug of choice. However, now $5 \%$ bacteria are resistant to more than one antibiotic. Antibiotic resistance is a burden to the entire world, in U. S. alone 99,000 deaths occur annually (Aslam et al. 2018). Antibiotics are also used as growth promoters in animal husbandry (poultry feeds) and agriculture. This has probably led to increase in the number of infections caused by multidrug resistant bacteria which is a threat to human life according to recent world economic global risk report (CDC, 2019). Antibiotic resistant organisms are a result of human activity is controversial because such organisms have been isolated from the environments devoid of human intrusions supporting the hypothesis that antibiotic resistance is an ancient and genetically rich natural phenomenon (D'Costa et al. 2011).

The bacterial evolution studied by phenotypic tests like metabolic activity, drug resistance or genetic tests like variations in chromosomal structure or individual gene sequence were useful but had limitations as they gave information of very small variations and not whole picture or resolution. The recent advances in whole genome sequencing and comparing it with the previously sequenced genomes helps to study evolutionary, resistant, pathogenicity as well as virulent mechanism of bacterial pathogen. Advances in 
sequencing technologies helps to capture sequence variation for each sample under study, providing the potential to detect all changes at all positions in the genome from single nucleotide changes to largescale insertions and deletions (Bryant et al. 2012; Pu et al. 2019). The WGS gives most precise structural details and definitive phylogenetic relationships thus helping researchers in understanding molecular mechanisms of antimicrobial resistance (Hendriksen et al. 2019) a vital step for this is detection of resistant determinants like single nucleotide polymorphism (SNP), Insertions or Deletions (INDELS) and frame shift mutations. SNP may produce synonymous mutation (SSNP) where an amino acid substitution may be silent causing no change in the encoded protein or nonsynonymous mutation (nSNP) where the amino acid may change the function of a protein. INDELS and frameshifts may alter a protein function significantly or a stop codon may halt a function abruptly. A point mutation in nonprotein coding region may affect regulatory element (Bryant et al. 2012).

In the present study, we addressed two questions. First, how many repetitions of ciprofloxacin exposure would result in resistant strain? Secondly what kind of genotypic change would lead to resistance?

\section{Materials And Methods}

\section{Microbial Culture}

S. Typhimurium MTCC 98 was purchased from Microbial Type Culture Collection Chandigarh, India.

\section{Media and antibiotics}

All the following media, antibiotics and antibiotic discs were purchased from Hi Media Laboratories, Mumbai, India: Muller Hinton Broth (MHB), Muller Hinton Agar (MHA), Luria Broth (LB), Luria agar (LA), Xylose-Lysine Deoxycholate agar (XLDA), ciprofloxacin, ampicillin, tetracycline, chloramphenicol, kanamycin powders. Discs of ciprofloxacin $(10 \mu \mathrm{g} / \mathrm{ml})$, ampicillin $(10 \mu \mathrm{g} / \mathrm{ml})$, tetracycline $(30 \mu \mathrm{g} / \mathrm{ml})$, chloramphenicol $(30 \mu \mathrm{g} / \mathrm{ml})$, kanamycin $30 \mu \mathrm{g} / \mathrm{ml})$, enrofloxacin $(10 \mu \mathrm{g} / \mathrm{ml})$, gatifloxacin $(5 \mu \mathrm{g} / \mathrm{ml})$, lomefloxacin $(5 \mu \mathrm{g} / \mathrm{ml})$, ofloxacin $(2 \mu \mathrm{g} / \mathrm{ml})$, levofloxacin $(5 \mu \mathrm{g} / \mathrm{ml})$, sparfloxacin $(5 \mu \mathrm{g} / \mathrm{ml})$

\section{Minimum Inhibitory Concentration (MIC)}

MIC of the cultures was determined according to the guidelines provided by Clinical and Laboratories Standards Institute (CLSI) by microdilution method (CLSI, 2020). The overnight culture (approximately $1 \mathrm{x}$ $10^{8} \mathrm{cfu} / \mathrm{ml}$ ) was diluted in the fresh MHB (1:10). To the first well of 96-well microtiter plate (Corning, US) $10 \mu \mathrm{l}$ of culture and 1:10 diluted stock concentration of ciprofloxacin $(10 \mathrm{mg} / \mathrm{ml})$ were added. The volume of the well was made $200 \mu \mathrm{l}$ using MHB. To the remaining wells in a row $100 \mu \mathrm{l}$ of MHB was added, solution from first lane was diluted twofold to proceeding wells. The plates were incubated at $37^{\circ} \mathrm{C}$ for 18 hrs. The lowest dilution of ciprofloxacin that showed no visible growth was noted as Minimal Inhibitory Concentration (MIC).

\section{Antibiotic Susceptibility Test (AST)}


AST was performed as per standard protocol CLSI (2020) method. Briefly, an overnight culture was diluted to obtain McFarland standard of 0.5 at $600 \mathrm{~nm}$. This was spread plated on previously prepared MHA plates. The plates were dried for $5 \mathrm{~min}$. The required antibiotic discs ciprofloxacin $(10 \mu \mathrm{g} / \mathrm{ml})$, ampicillin $(10 \mu \mathrm{g} / \mathrm{ml})$, tetracycline $(30 \mu \mathrm{g} / \mathrm{ml})$, chloramphenicol $(30 \mu \mathrm{g} / \mathrm{ml})$, kanamycin $30 \mu \mathrm{g} / \mathrm{ml})$. enrofloxacin $(10 \mu \mathrm{g} / \mathrm{ml})$, gatifloxacin $(5 \mu \mathrm{g} / \mathrm{ml})$, lomefloxacin $(5 \mu \mathrm{g} / \mathrm{ml})$, ofloxacin $(2 \mu \mathrm{g} / \mathrm{ml})$, levofloxacin $(5 \mu \mathrm{g} / \mathrm{ml})$, sparfloxacin $(5 \mu \mathrm{g} / \mathrm{ml})$ were placed on the bacterial lawn and incubated at $37^{\circ} \mathrm{C}$ for $18 \mathrm{hrs}$. The zone of inhibition $(\mathrm{mm})$ was measured and results interpreted as per the chart provided by CLSI (2020).

\section{Laboratory development of ciprofloxacin resistance}

The overnight culture was inoculated in LB for 3 hrs. to obtain a logarithmic phase (approx. $4 \mathrm{x}$ $\left.10^{4} \mathrm{cfu} / \mathrm{ml}\right)$. The $5 \mu \mathrm{l}$ of culture was then added to a fresh LB $(5 \mathrm{ml})$ containing ciprofloxacin 10 times the MIC and allowed to grow for $18 \mathrm{hrs}$ at $140 \mathrm{rpm}$. The $5 \mu$ inoculum was then suspended in fresh LB; allowed to grow for $3 \mathrm{hrs}$ and again exposed to ciprofloxacin (10X MIC) as explained earlier. After each cycle MIC was determined. After 20 cycles of growth and ciprofloxacin exposure the culture was plated on LA plate with ciprofloxacin $(10 \mu \mathrm{g} / \mathrm{ml})$ incubated at $37^{\circ} \mathrm{C}$ for $18 \mathrm{hrs}$. Three clones were selected and processed for phenotypic and genotypic analysis.

\section{Stress experiments}

\section{Heat stress}

An overnight culture supplemented with ciprofloxacin was washed with saline and inoculated in fresh medium. These were heated at different temperatures $\left(45^{\circ} \mathrm{C}, 50^{\circ} \mathrm{C}, 55^{\circ} \mathrm{C} \& 58^{\circ} \mathrm{C}\right)$ for $10 \mathrm{~min}$ and immediately appropriate dilutions were spread plated on LA plates. The colony forming units $(\mathrm{cfu} / \mathrm{ml})$ was determined after incubation at $37^{\circ} \mathrm{C}$ for $18 \mathrm{hrs}$.

\section{Osmotic stress}

An overnight culture supplemented with ciprofloxacin was washed with saline and inoculated in fresh LB containing different concentration of $\mathrm{NaCl}(3,5,6,12 \& 15 \%)$. These were then incubated at $37^{\circ} \mathrm{C}$ at 160 rpm. After overnight incubation the appropriate dilutions were spread plated on LA plates. The colony forming units (cfu/ml) was determined after incubation at $37^{\circ} \mathrm{C}$ for $18 \mathrm{hrs}$.

\section{Determination of lag phase}

A 96-well microtiter plate was used for the experiment. A logarithmic phase culture (0.5 at OD $600 \mathrm{~nm})$ was added (5 $5 \mathrm{l})$ to $195 \mu \mathrm{l}$ of LB. The growth analysis was carried out at an absorbance of $600 \mathrm{~nm}$ on Synergy H1 microplate reader, Biotek (Winooski, Vermont, U.S.A). A medium shaking speed (120 rpm) was used and optical densities recorded after every $20 \mathrm{~min}$ for $20 \mathrm{hrs}$ at $37^{\circ} \mathrm{C}$.

\section{Whole Genome Sequencing (WGS)}


The whole genome sequencing was carried using Illumina HiSeq 4000 sequencer (Illumina, San Diego, USA), following manufacturer's instructions at Genotypic Technologies Pvt. Ltd. Bangalore, India. The sequencing quality was assessed using FastQC v0.11.8 software. The adapter clipped, high quality reads were aligned to the genome sequence of S. Typhimurium, strain-LT2 using Bowtie2 (Langmead and Salzberg 2012) and variant calling was done using Samtools 1.3 (Li et al. 2009). The significant variants were annotated and consensus sequence obtained. The data obtained from the sequencing analysis was analyzed in Jupyter notebook with python 3.7 backend in the Anaconda package. The Circos plot was made by compiling the chromosome coordinates and annotates mutations and plotting the data with circos-0.69-9 (Krzywinski et al. 2009). The genes with non-synonymous mutations were submitted to the Panther database public server (Mi et al. 2019) and DAVID database (Huang et al. 2009) for gene function classification. The protein-protein interaction of the DNA metabolic genes and resistance genes were obtained from STRING v11 public server (Szklarczyk et al. 2019). 3D structure of gyrA was obtained by intensive modelling at PHYRE 2 server and string analysis performed using string database (https://string-db.org/). We scanned the genome of the variants for presence of resistance genes at the Comprehensive Antibiotic Resistance Database (Alcock et al 2020). Details of software's used are provided in Supplementary Information Materials and Methods.

\section{Results}

\section{Development of antibiotic resistance}

S. Tyhimurium is sensitive to ciprofloxacin (MIC $0.05 \mu \mathrm{g} / \mathrm{ml}$ ). When $S$. Typhimurium was exposed to 10 times MIC concentrations of ciprofloxacin, $99 \%$ of the population was eliminated. The remaining $1 \%$ of the population was again treated with $10 \mathrm{X}$ MIC concentration of ciprofloxacin. This ciprofloxacin exposure was repeated up to 20 cycles. In each cycle MIC of the S. Typhimurium was measured. The ciprofloxacin resistance was increased up to 100 -fold (MIC $5 \mu \mathrm{g} / \mathrm{ml}$ ) after 10 th cycle. There was no further increase in antibiotic resistance up to 20th cycle. After 20th exposure 3 resistant $S$. Typhimurium clones were selected for detailed evaluation. These clones were designated as $A, B$ and $C$ and were characterized in detail with respect to phenotype and genotype.

\section{Phenotypic Characterization}

The clones exhibited similar characteristics as wild type, both were Gram negative, lactose nonfermenters and showed typical dark centred colonies on selective media (XLD, HEA, BSA). However, the clones formed smaller colony on LB $(0.7 \pm 0.5 \mathrm{~mm})$ as compared to wild type $(2 \pm 0.5 \mathrm{~mm})$. The clones had significantly longer lag phase (70 min) as compared to wild type (20 min) (Fig. 1). A, B and C were also resistant to diverse antibiotics belonging to different classes of antibiotics like beta-lactam, aminoglycosides, chloramphenicol and tetracycline along with fluoroquinolones. This is indicated by lower or no zone of inhibition of the clones as compared to the wild type (Table 1). However, the clones ( $A, B$ and $C$ ) were more sensitive to heat and osmotic stress than the wild type. This can be observed by lower count of the clones viz $3.18 \mathrm{cfu} / \mathrm{ml}$ and $2.64 \mathrm{cfu} / \mathrm{ml}$ to heat and osmotic stress respectively, while, 
the wild type strain had higher counts of $4.03 \mathrm{cfu} / \mathrm{ml}$ and $4.96 \mathrm{cfu} / \mathrm{ml}$ to the heat and osmotic stress respectively (Table 1 ).

Table 1

Phenotypic characteristics of wild type and Clones (A, B and C)

\begin{tabular}{|lll|}
\hline Characteristics & Wild type & Clones (A, B and C) * \\
\hline Colony size & $2.0 \mathrm{~mm}$ & $0.7 \mathrm{~mm}$ \\
\hline MIC & $0.05 \mu \mathrm{g} / \mathrm{ml}$ & $5.0 \mu \mathrm{g} / \mathrm{ml}$ \\
\hline Lag phase & $20 \mathrm{~min}$. & $70 \mathrm{~min}$. \\
\hline Antibiotic sensitivity & Sensitive & Resistant to different classes of antibiotics ${ }^{\#}$ \\
\hline Heat stress ( $\left.55{ }^{\circ} \mathrm{C}-10 \mathrm{~min}\right)$ & $4.03 \mathrm{cfu} / \mathrm{ml}$ & $3.18 \mathrm{cfu} / \mathrm{ml}$ \\
\hline Osmotic stress (15\% NaCl overnight) & $4.96 \mathrm{cfu} / \mathrm{ml}$ & $2.64 \mathrm{cfu} / \mathrm{ml}$ \\
\hline $\begin{array}{l}\text { *There was no significant difference between the three clones with respect to phenotypic } \\
\text { characteristics and stress tolerance }\end{array}$ & \\
\hline $\begin{array}{l}\text { \# All the three clones were resistant to aminoglycoside, beta-lactam, chloramphenicol, tetracycline } \\
\text { along with fluoroquinolone }\end{array}$ & \\
\hline
\end{tabular}

\section{Genotypic Characterization}

The whole genomic sequencing of three ciprofloxacin resistant clones of $S$. Typhimurium and parent strain was carried out. The sequences of all the clones were matched with the parent sequence and amongst themselves. All three clones had similar mutations suggesting that they originated from one founder strain. Clones $A$ and $C$ were closely related with only $0.2 \%$ variation observed. However, $A$ and $B$ diverged at some point and difference of $4.2 \%$ was observed. While, the difference between $B$ and $C$ was 3.8\% (Supplementary Information Table S1).

The detailed genomic analysis of clone A was carried out and the differences between clone A and B are discussed in a relevant section. The genome analysis revealed 40858 single nucleotide polymorphisms (SNPS) and 113 INDELS distributed across the genome. There are total 4489 genes annotated in $S$. Tyhimurium LT2. Total 3836 genes were mutated; that is $95 \%$ of $S$. Typhimurium genome was mutated. However, majority of these mutations observed (15344 mutations) were synonymous mutations. A total of $23.13 \%$ genes showed nonsynonymous mutations. Many genes showed multiple mutations comprised of both synonymous and non-synonymous mutations (Supplementary Information Table S2). However, in these genes number of synonymous mutations was higher than non-synonymous mutations. This can be illustrated with one example. The gene $\mathrm{met} H$ had total 59 mutations. Out of 61 mutations 57 were synonymous mutations and 4 mutations were non-synonymous. The mutations were also observed in intergenic regions. Total 4418 mutations were observed in intergenic regions. Current report is therefore 
the first report of high SNPs in a single isolate. The mutation caused by ciprofloxacin exposure was random. The Circos plot indicates uniform distribution of the SNPs and INDELS across the genome (Fig. 2A). One interesting observation was, the plasmid of $90 \mathrm{~kb}$ had only two mutations which is significantly less than the genome. Transition mutations were 4-fold compared to transversion mutations (Fig. 2B).

\section{Highly mutated genes and location of mutations}

The genes $m e t H, m u k B, g c v P$ and $g / t B$ showed more than 45 mutations per gene. The interesting observation was gene like STM4261 (siiE) the largest gene of 16680 bp responsible for putative inner membrane protein showed highest number (159) of SNP as well as nSNPs (58). However, gene STM2690 of length $1409 \mathrm{bp}$ responsible for efflux transmembrane transport protein had 10 SNPs but number of nSNPs were only 3 . Another important gene muk $B(4467 \mathrm{bp})$ involved in chromosomal condensation, segregation and cell cycle progression was mutated with 55 SNPs, it had two nSNPs (D294E andW945G). The location of SNPs in the present study is not specific but covers the entire chromosome as observed for the genes $d n a E, g y r A$, iroC, metH and $r p o B$ (Fig. 3) point mutations which is against the observation that chromosomal location determines the type of mutation (Hudson et al. 2002).

\section{The most probable genes that could contribute to antibiotic resistance}

The mutants had 23 SNPs in gyrA with the common S83F nSNP. The 3D structure of gyrA shows Ser83 is replaced by phenylalanine, the most common cause of quinolone resistance. Here a small polar amino acid is replaced by bulky phenylalanine (Fig. 4) which creates a narrow rigid pocket for DNA. Total 33sSNP were observed in gyrB. Besides gyrA we found nonsynonymous mutations in par $\mathrm{C}$ and polA also we report 36SNPs in parC (with 3 nSNP T620A, A469S, A57S) which are different than those noted by the earlier researchers as parC mutations are rare in Gram negative organisms. A total of 14 SNP with a single nSNP (nSNP P571S) in parE was observed though parE mutation is seldom responsible for ciprofloxacin resistance. The genes acrA and $a c r B$ encoding efflux pump proteins were mutated with 14 and 26 SNPs respectively, but only one nSNP at T270S was observed in acrA. While, the outer membrane channel protein encoding gene tolC had total twelve sSNPs and single nSNP at the position D457E. Moreover, two putative efflux pumps encoded by gene $y e g \mathrm{~N}$ and yegO also showed 41 and 45 SNPs with 7 and 2 nSNPs respectively. Further the efflux pump regulators marR, acrR and, soxR genes had 2, 2 and 3 sSNPs but no non-synonymous mutations probably, showing efflux activity was important in present study which needs to be further explored. Genes encoding outer membrane protein OmpA and porins OmpC, OmpF show 5, 9 and 7 sSNPs with single nSNPs in ompA and ompF respectively. The transcription and translation machinery also play the role in antibiotic resistance. The clone A showed 52 SSNPs in rpoC distributed across the gene except a narrow band of $400 \mathrm{bp}$ devoid of mutation while a single nSNP and 32 sSNPs were observed in $r p o B$ gene probably indicating role of these genes in resistance as noted by other researchers. The gene $\mathrm{metH}(3771 \mathrm{bp}$ ) is responsible for iron transporter for ABC efflux pump. This gene had 4 nSNPs within 61 total mutations distributed over the entire gene but genes sulA, yggX important in a negative regulator of cell division and iron-sulphur binding and oxidative stress respectively showed only single sSNP each. The other genes with high mutations were $s b c C$, recD 
with 48 and 26 SNPs respectively and 7nSNPs; involved in SOS repair pathways. Two more genes iroC encoding $A B C$ mediated involved in virulence and polA encoding an enzyme involved in DNA repair and proof-reading activity are highly mutated with 48 and 27 SNPs including 13 and 3 nSNPs respectively. String analysis shows a relationship between $s b c \mathrm{C}, r e c \mathrm{D}$, gyrA and polA (Supplementary Information Fig. S1).

\section{Functional analysis of the mutants}

In most of the antibiotic resistant mutants, altered metabolism has been observed in DNA repair and replication pathways, oxidative stress management pathways and cell wall and cell membrane synthesis pathways. In the current study, we observed multiple proteins in these pathways were altered by nonsynonymous mutations. Few of the important pathways are highlighted. Both Panther and David databases showed following pathways were mutated (Fig. 5). In transcription regulation 61 to $64 \%$ genes analysed had mutation. The prominent genes found to be mutated were $p r p R, \operatorname{ssr} B, \operatorname{arc} B, r p o B$ and tor $B$. Transmembrane transport mutations are common in antibiotic resistant strains. We observed 40 to $53 \%$ gene in this category were mutated (yajR, emrB, yeaN, ydhC, $m d f A$ ). Mutations were also observed in genes fimA, fimH, fliD encoding proteins for cell adhesion; genes sopA, sopB, sseA, pipB encoding for pathogenesis and genes fimC, stiC, stiB responsible for pilus assembly pathways. Details of the genes described in the text with the position of nSNPs are given in Supplementary Information Table S2

\section{Comparison of mutations amongst three clones (A, B and C)}

All the three clones developed maximum resistance after 10 cycles but they acquired this at different cyclic exposure (Supplementary Information Fig. S2). A gradual increase in MIC to $1.25 \mu \mathrm{g} / \mathrm{ml}$ in 4th cycle and $2.5 \mu \mathrm{g} / \mathrm{ml}$ in 6th cycle respectively was observed in clone $A$. Clone B showed sudden increase within 2 cycles to $2.5 \mu \mathrm{g} / \mathrm{ml}$ and reached maximum of $5 \mu \mathrm{g} / \mathrm{ml}$ after 10 cycles while clone $C$ showed sudden increase to $5 \mu \mathrm{g} / \mathrm{ml}$ after 4 cycles. This shows clone $B$ acquired resistance earlier but is less resistant to ciprofloxacin as it shows a bigger zone of inhibition compared to clones $A$ and $C$ (Table 2). It is also less resistant to chloramphenicol, tetracycline and co-trimethazole (SF 1), However, WGS shows it has higher SNPs and INDELs (Supplementary Information Table 1). It shows additional nSNP at T67S in parC and $r p o \mathrm{C}$ at $\mathrm{P} 1026 \mathrm{~S}$ amongst the genes important in antibiotic resistance which needs to be studied further. 
Table 2

Antibiotic sensitivities of Wild type and Clones A, B and C to different antibiotics

\begin{tabular}{|c|c|c|c|c|}
\hline \multirow[t]{2}{*}{ Antibiotics } & \multicolumn{4}{|c|}{ Zone of inhibition (mm) } \\
\hline & Wild & A & B & C \\
\hline Ampicillin $(10 \mu \mathrm{g} / \mathrm{ml})$ & 8.3 & nil & nil & nil \\
\hline Chloramphenicol $(30 \mu \mathrm{g} / \mathrm{ml})$ & 25 & nil & nil & nil \\
\hline Ciprofloxacin $(10 \mu \mathrm{g} / \mathrm{ml})$ & 36.3 & 10 & 15.6 & 11.6 \\
\hline Kanamycin $(30 \mu \mathrm{g} / \mathrm{ml})$ & 17 & nil & nil & nil \\
\hline Tetracycline $(30 \mu \mathrm{g} / \mathrm{ml})$ & 23 & 8 & 8 & 8 \\
\hline Enrofloxacin $(10 \mu \mathrm{g} / \mathrm{ml})$ & 27 & 13 & 13 & 16 \\
\hline Gatifloxacin $(5 \mu \mathrm{g} / \mathrm{ml})$ & 31 & 10 & 10 & 15 \\
\hline Lomefloxacin $(5 \mu \mathrm{g} / \mathrm{ml})$ & 30 & 13 & 13 & 18 \\
\hline Oflaxcin $(2 \mu \mathrm{g} / \mathrm{ml})$ & 32 & 10 & 10 & 20 \\
\hline Levofloxacin $(5$ g/ml) & 36 & 10 & 13 & 16 \\
\hline Sparfloxacin $(5 \mu \mathrm{g} / \mathrm{ml})$ & 31 & 10 & 10 & 17 \\
\hline \multicolumn{5}{|c|}{$\begin{array}{l}\text { Overnight culture was diluted to obtain } 5 \times 10^{5} \mathrm{cfu} / \mathrm{ml} \text { of culture. It was spread plated on MHA plates. } \\
\text { Antibiotic discs of diffwerent concentration of antibiotics were placed on the plates and incubated at } \\
37^{\circ} \mathrm{C} \text { for } 18 \mathrm{hrs} \text {. Zone of inhibition measured and compared with standard charts provides by CSLI } \\
(2020)\end{array}$} \\
\hline
\end{tabular}

\section{Discussion}

Many approaches are followed to understand evolution of antibiotic resistance. The Adaptive Laboratory Evolution ( ALE) is used in most of the studies (Oz et al. 2014; Munck et al. 2014; Kim et al. 2018). In ALE, two approaches were followed, first, incremental increase in the antibiotic concentration and second method stepwise exposure to antibiotics (Jahn et al. 2017). In the current study, stepwise antibiotic exposure was used. The ciprofloxacin was the choice of antibiotic. One of the most effective antibiotics used to treat $P$. aeruginosa infections is the second-generation fluoroquinolone, ciprofloxacin; introduced in 1987, ciprofloxacin proved so effective at treating an infection that it rapidly joined the WHO list of medicines essential for basic healthcare (Wise et al. 1983). It has a broad spectrum of action with good tissue penetration, oral absorption and favourable pharmacokinetics, making it ideal for the treatment of a wide range of infections. Crucially, the presence of the cyclopropane moiety on the $\mathrm{N}$ atom of the heterocycle in ciprofloxacin increases its activity (compared with first generation fluoroquinolones such as norfloxacin) against $P$. aeruginosa by a factor of four (Jedrey et al. 2018). The second reason for the use of ciprofloxacin is its ability to induce more mutations by interfering with DNA replication and repair mechanism (Maxwell et al. 2015; Bush et al. 2020). Suzuki et al (2014), suggested that, the antibiotic 
resistance development in a gradual manner is unlikely. Evolution often happens in spurts (Neher 2013). In our experiments we observed that, the resistance increases by 25 and 50 folds after, 4 and 6 cycles respectively in clone $A$. After 10th cycle 100 -fold increase in resistance was observed and this is the plateau of the resistance. P. aeruginosa when exposed to constant sub-inhibitory concentrations or increasing concentration of quaternary ammonium compounds showed a plateau in MIC after 10 cycles leading to ciprofloxacin resistance (Kim et al. 2018; Voumard et al. 2020). This is probably due to regrowth of bacteria tolerant to antibiotic after initial kill of $90-95 \%$ population as we have noted the MIC after the growth up to approximately $10^{7}$ cells. These tolerant bacteria remove ciprofloxacin by efflux, grows and also maintains the structure of outer membrane showing involvement of different mechanisms simultaneously i.e., initial stress response of the surviving population. The tolerant bacteria probably pump out ciprofloxacin by efflux and grow (Moen et al. 2012). This shows higher concentrations of antibiotics present in the broth (10X MIC) as in our case or in industrial sewage or clinical environment are more prone to antibiotic resistant bacteria than the sub-inhibitory or lower concentrations used in food processing, animal husbandry or veterinary conditions as pointed out by Gullberg et al (2011). Eagle (1948) noted that bacteria or fungi surviving above its bactericidal concentration had improved survival leading to antibiotic resistances. Survival of Staphylococci 1000-fold above its MIC in penicillin was also observed (Kirby 1945; Prasetyoputri et al. 2019). However, MIC does not give any phenotypic or genotypic knowledge of the bacterium therefore; we have characterized the mutants $(A, B, C)$. The observations from this study and previous study clearly suggest the randomness of the event. The founder mutation will lead to multiple mutations. However, in the current study we have not sequenced the survivors of each cycle to screen for mutations. Our objective was to know what is the maximum resistance that could be achieved in ALE experiments.

The exposure of the wild type strain to ciprofloxacin 10 times its MIC showed lower growth rate as indicated with an increased lag phase (70 $\mathrm{min}$ ) of the mutant strain (Fig. 1). This delay in growth probably is the strategy of bacteria to remain dormant, prevent itself from the harms of the antibiotic and prepare for the reproduction. The bacteria first become tolerant to in lag phase and this "tolerance by lag "leads to antibiotic resistance. (Fridman et al. 2014; Li et al. 2016). Fridman et al (2014) observed on exposing the Escherichia coli strain to ampicillin for 3, 5 and cycles increased the lag to 3.5, 6 and $10 \mathrm{hrs}$ respectively; therefore, authors presume lag phase is optimized to tolerate antibiotic stress. $S$. Enteritidis mutants (SE-M1, SE-M2), which presented reduced susceptibility to ciprofloxacin, exhibited the same growth as the parental strain; however, the mutants that acquired resistance had longer lag phases than the parental strain and did not reach the same cell density in the stationary phase. On exposure to ciprofloxacin a longer lag phase of approximately $12 \mathrm{hrs}$ was observed in S. Enteritidis and $S$. Typhimurium mutants (Zhang et al. 2017).

All three mutants in the current study were sensitive to heat and osmotic stress (Table 1). Cellular responses, antimicrobial exposure, and other growth-compromising stresses, have all been linked to the development of antimicrobial resistance in Gram-negative bacteria resulting from the stimulation of protective changes to cell physiology, activation of resistance mechanisms, and induction of resistance 
mutations (Poole 2012). However, these mutants were resistant to different antibiotics like ampicillin, kanamycin, tetracycline and chloramphenicol which belong to different classes of antibiotics viz: betalactam, aminoglycoside, tetracyclines and chloramphenicol along with fluoroquinolones having different modes of action. The resistance to different generations of ciprofloxacin was also observed (Table 2). Thus, ciprofloxacin induced antibiotic resistance cause multidrug resistance. The antibiotics probably have common targets or common mechanism to develop resistance. Jahn et al (2017) have shown that E. coli can adapt to resistant mutation in different antibiotics like amikacin, piperacillin and tetracycline irrespective of method of selection or adaptation. Recently E. coli and S. Typhimurium mutants resistant to tetracycline (tigecycline), beta-lactam (mecillinam) and antimicrobial peptide (protamine) showed increased sensitivity to antibiotic nitrofurantoin under laboratory condition (Roemhild et al. 2020).

Colony size of bacteria is phenotype variation to study genetic diversity and intermediate exposure to antibiotics may cause small size colonies (Lee et al. 2018). Small colony size and long lag phase has been associated with exposure to aminoglycoside stresses in $P$. aeruginosa (Wei et al. 2011). In clinical isolates of $S$. aureus small colony variants have been linked to antibiotic resistant infections (Cao et al. 2017). The bacteria may show phenotypic switching of colony size shape or cell morphology to adapt to a hostile environment and this needs to be studied further. In this study small colony size has probably helped in ciprofloxacin resistance adaptation.

After 20 cycles of exposure to ciprofloxacin, 3 clones were selected for WGS. The objective was to understand, what mutations led to resistance? Second objective was to find out whether all the clones have originated from the same parental strain or each evolved independently? There could be multiple evolutionary pathways which lead to each clone. However, sequencing each clone and its ancestor was beyond the scope of this work. The whole genome comparison of three clones suggests that, they are derived from a single parental strain. This strain may have a founder mutation, probably in gyrase or in DNA repair pathway. In an earlier study on evolution of resistance in $S$. aureus after 22 days of antibiotic exposure (trimethoprim, ciprofloxacin and neomycin) WGS was carried out. They sequenced 120 clones and found that treatments with alternating antibiotics changed the spectrum of resistance mutations. These genetic constraints affected the rate of evolution of mutations associated with the cross resistance amongst the drugs (Kim et al. 2014). E. coli on exposure to amikacin, tetracycline and piperacillin at the end of the study 96 clones were sequenced and found that cross resistance is similar in the two approaches used in the study leading to similar phenotypic and genotypic changes (Jahn et al. 2017).

We report for the first-time approximately 40,000 SNPs in a single isolate on cyclic exposure to ciprofloxacin. No such report on single isolate is observed in literature. Ten clinical Pseudomonas aeruginosa mutants resistant to amikacin showed 18,876 nSNPs while, 81 nSNPs were identified in pipercellin resistant isolates (Ramanathan et al. 2017). Similarly, in ten clinical isolates of Acinetobacter baumanii 11,387 SNPs in the coding region, 42 INDELS and 33 antibiotic related genes were observed. Total 74,713 SNPs have been reported in 60 isolates of MDR E. coli recently from India (Pu et al. 2019; Ragupathi et al. 2020). The clone A showed 113 INDELS. Out of these 10 (9 insertions and 1 deletion) 
were in the coding region while others in the non-coding regions. These were present in genes not essential for antibiotic resistance. In Mycobacterium tuberculosis short indels are shown to cause antibiotic resistance upon disruption which are not only dispensable and important in highly resistant outbreak but also important in evolution (Godfroid et al. 2020). WGS of 25 clinical isolates of pneumococcal strains from children showed different drug resistant profiles ranging from 131-171 indels and SNPs in the range 16,103-28,128 with different drug resistant profiles (Pan et al. 2018) however, the contribution of indels in antibiotic evolution is poorly understood.

All clones in the present study showed $80 \%$ transitions and $20 \%$ transversions. In earlier studies also, similar ratio of 1:4 ratio of transversion: transition was observed in multidrug resistant $E$. coli, Mycobacterium tuberculosis and human genome (Guo et al. 2017; Payne et al. 2019; Ragupathi et al. 2020). In a previous study, it has been shown that, fluoroquinolone (norfloxacin) antibiotics disrupt DNA repair pathways. This impaired mismatch repair pathway may also contribute to skewed ratios of transition to transversion. (Jørgensen et al. 2013). The widespread biasness observed in transition: transversion ratios are unknown. Two main hypotheses to explain this phenomenon are; first the mutational hypothesis which shows there is transitional biasness and higher transitional rate in coding and noncoding regions therefore transition mutational rates of polymerases are higher than transversion rates. $[49,50]$. The second selective hypothesis states that nonsynonymous transitions conserve important biochemical properties of original amino acids therefore natural selection does not favour transversions (Vogel and Kopun 1977; Miyata et al. 1979; Zhang 2000; Lyons and Lauring 2017). The synonymous mutations do not affect the encoded amino acid and therefore, have no role in adaptation or fitness of a bacterial cell. However, laboratory evolved populations of $P$. fluorescence and S. Typhimurium have shown increased fitness due to synonymous mutation (Lind et al. 2010; Bailey et al. 2014). This shows our knowledge about gene function and their role in bacterial genome evolution is still scarce as pointed out by Bryant et al (2012).

In all three clones the ciprofloxacin induced mutation frequency across the genome was uniform except the plasmid ( $p s L T$ ) (Fig. 2A) There are many genes in the current study had large number of SNPs. However, the number of mutations per 1000 bp was uniform across the genome. Hence, bigger genes like STM_4261 (siiE) have more mutations compared smaller genes like marR which have fewer mutations. However, there are many reports of biased mutation frequency concentrated in certain region of genome. In a recent study Foster et al (2013), reported that mutations are not randomly distributed along the chromosome rather, mutations fall in a wave-like pattern that is repeated in an almost exact mirror image in the two separately replicated halves (replicores) of the E. coli chromosome (Martincorena et al. 2012; Foster et al. 2013). Mutation is difficult to study because it is a highly noisy process and because it affects variation in a manner that is highly entangled with the effects of natural selection. To characterize the effects of mutation, we need to acknowledge these complications and find creative ways to address them. Future studies will undoubtedly take advantage of the increasing ability to examine variation at the whole-genome level to reveal much more about mutation and how it acts as an engine of evolution in bacteria (Hershberg 2015). 
Mutations were uniformly distributed across the genome. Therefore, multiple metabolic pathways are affected. Since, selection was based on the survival, all the three clones were able to grow on rich medium albeit slow. Most of the metabolic pathways are functioning or alternative pathways are functioning to keep the metabolism going. However, compared to wild type the mutants grew slow and formed small colony. Survival and growth in high antibiotic concentration are the result of contribution of multiple adjustments in many metabolic pathways. The mutant should have corrupted target or targets that are not affected by antibiotic. All three mutants had mutations in DNA replication and repair pathways. The increase in MIC of ciprofloxacin is known to be by alterations in primary and secondary drug targets gyrA, gyrB the DNA gyrases or parC, parE the topoisomerases and regulator genes of efflux pumps like (marR, acrR and soxR); recently RNA polymerases $r p o \mathrm{~B}, r p o \mathrm{C}$ are also shown to improve import of ciprofloxacin in E. coli (Ricci et al. 2006; Pietsch et al. 2017). In previous studies it has been shown that, a single mutation in gyrA at S83 orA87 was observed in fluoroquinolone resistant Salmonella from human and animal origin. Similarly, resistance linking gyrB mutation at (S464T) and mutations at $\mathrm{S} 80$ or G78 of parC has been reported in ciprofloxacin resistant Salmonella (Chen et al. 2007). The second possibility is pumping out the antibiotic or restriction of antibiotic entering into the cell. Active efflux and decrease in drug permeability also contribute to resistance to many antibiotics including fluoroquinolones in clinical isolates of Salmonella, E. coli, Pseudomonas etc; (Toprak et al. 2011; Redgrave et al. 2014) acrAB-to/C efflux pump is the prominent one in S. Typhimurium. This pump consists of periplasmic accessory protein AcrA, transporter inner membrane protein AcrB and outer membrane channel protein (TolC). The mutants had multiple mutations in transporter proteins. It has been shown that ciprofloxacin affects cell viability by causing oxidative stress. We observed at least 45 genes involved in oxidative stress were mutated. However, none of these mutations were biologically tested for their contribution for the antibiotic resistance. The antibiotic resistance observed in the current study may be cumulative effect of mutations in many pathways or mutation in few genes. This needs further validation by detailed physiological studies. (Toprak et al. 2011; Redgrave et al. 2014).

There are few observations which we were unable to explain. The $p S L T$ plasmid is a $90 \mathrm{~kb}$ native plasmid of $S$. Typhimurium. The entire plasmid had only two mutations. Since plasmid replication and maintenance are also carried out by genes on genome and expected similar mutation rate as on the main genome. The transformation experiments in $E$. coli have suggested that probably the mechanism of replication and maintenance is different in chromosome and plasmid. The other reason could be our dose of ciprofloxacin used in this study was high and cost of maintaining resistant plasmid will be ameliorated in evolution experiments as observed by Svara and Rankin, (2011).

The important shortcoming of the current study was, we were unable to track the mutations from the first cycle to 20th cycle of antibiotic exposure. This would have given an insight into possible origin of hypermutation phenotype. However, clones A, B and C all share more than $98 \%$ of the mutations; suggest that all have originated from one hyper mutant.

\section{Conclusion}


Ciprofloxacin resistant $S$. Typhimurium mutants could be spontaneously developed by exposing the sensitive organism repeatedly to a high concentration of ciprofloxacin for merely 10 cycles showing that antibiotic resistance has indeed increased due to anthropogenic activities. We propose mutation in gyrA is the first step towards ciprofloxacin resistance, while other extensive changes were selected to maintain homeostasis and hence no alteration to other stresses were noted. It is essential to further study the influence of the resistance on the different metabolic pathways which may contribute to its resistance to ciprofloxacin and cross resistance to other different classes of antibiotics. This study will help to further improve our knowledge on methods to overcome bacterial resistance and possibly development of new better antibiotics.

\section{Declarations}

\section{Availability of data and material}

The microbial culture is available with the corresponding author on request. The raw data of genome sequencing are available with GenBank with below mentioned accession numbers

Wild type S. Typhimurium and plasmid: local id: 1 \& 2; Accession no. CP074092 and CP074092

Clone A and plasmid: local id SO_8752_A_cip \& A_cip; Accession no. CP074094 and CP074095

Clone B and plasmid: local id SO_8752_Cip_2B \& B_cip_Plasmid; Accession no. CP074096 and CP074097

Clone C and plasmid SO_8752_C_Cip and C_cip_Plasmid; Accession no CP074098 and CP074099

\section{Code availability}

Not applicable

\section{Acknowledgement}

Authors acknowledge technical help provided by Mrs Vaishali Mahale

\section{Funding}

This research did not receive any specific grant from funding agencies in the public, commercial, or notfor-profit sectors.

\section{Author Contributions}

ASK: methodology, investigation, formal analysis, writing-original draft. AD: bioinformatic analysis, validation, reviewing and editing. RS: conceptualization, project administration, reviewing and editing of final draft.

\section{Ethics declaration}




\section{Conflict of interest}

The authors declare that they have no conflicts of interest

\section{Ethical approval}

Not applicable

\section{Consent to participate}

Not applicable

\section{References}

1. Alcock B, Rapheynya AR, Lau TTY, et al (2020) CARD 2020: antibiotic resistome surveillance with the comprehensive antibiotic resistance database. Nucleic Acids Res 48:D517-D525. https://doi.org/10.1093/nar/gkz935

2. Aslam B, Wang W, Arshad Ml, et al (2018) Antibiotic resistance: a rundown of a global crisis. Infect Drug Resist Volume 11:1645-1658. https://doi.org/10.2147/IDR.S173867

3. Bailey SF, Hinz A, Kassen R (2014) Adaptive synonymous mutations in an experimentally evolved Pseudomonas fluorescens population. Nat Commun 5:4076. https://doi.org/10.1038/ncomms5076

4. Bryant J, Chewapreecha C, Bentley SD (2012) Developing insights into the mechanisms of evolution of bacterial pathogens from whole-genome sequences. Future Microbiol 7:1283-1296. https://doi.org/10.2217/fmb.12.108

5. Bush NG, Diez-Santos I, Abbott LR, Maxwell A (2020) Quinolones: Mechanism, Lethality and Their Contributions to Antibiotic Resistance. Molecules 25:5662. https://doi.org/10.3390/molecules25235662

6. Cao S, Huseby DL, Brandis G, Hughes D (2017) Alternative Evolutionary Pathways for Drug-Resistant Small Colony Variant Mutants in <em>Staphylococcus aureus</em>. mBio 8:e00358-17. https://doi.org/10.1128/mBio.00358-17

7. CDC, 2019 https://www.cdc.gov/drugresistance/pdf/threats-report/nt-salmonella-508.pdf Accessed 22 November 2019 Drug-Resistant Nontyphoidal Salmonella. 2

8. Chen S, Cui S, McDermott PF, et al (2007) Contribution of Target Gene Mutations and Efflux to Decreased Susceptibility of Salmonella enterica Serovar Typhimurium to Fluoroquinolones and Other Antimicrobials. Antimicrob Agents Chemother 51:535-542. https://doi.org/10.1128/AAC.00600-06

9. CLSI Clinical and laboratory Standards Institute. (2020) Performance Standards for Antimicrobial Susceptibility Testing. 30th ed. CLSI supplement M100. Wayne, PA

10. D'Costa VM, King CE, Kalan L, et al (2011) Antibiotic resistance is ancient. Nature 477:457-461. https://doi.org/10.1038/nature10388 
11. Foster PL, Hanson AJ, Lee H, et al (2013) On the Mutational Topology of the Bacterial Genome. G3 GenesGenomesGenetics 3:399-407. https://doi.org/10.1534/g3.112.005355

12. Fridman $\mathrm{O}$, Goldberg A, Ronin I, et al (2014) Optimization of lag time underlies antibiotic tolerance in evolved bacterial populations. Nature 513:418-421. https://doi.org/10.1038/nature13469

13. Godfroid M, Dagan T, Merker M, et al (2020) Insertion and deletion evolution reflects antibiotics selection pressure in a Mycobacterium tuberculosis outbreak. PLOS Pathog 16:e1008357. https://doi.org/10.1371/journal.ppat.1008357

14. Guo C, McDowell IC, Nodzenski M, et al (2017) Transversions have larger regulatory effects than transitions. BMC Genomics 18:394. https://doi.org/10.1186/s12864-017-3785-4

15. Hendriksen RS, Bortolaia V, Tate H, et al (2019) Using Genomics to Track Global Antimicrobial Resistance. Front Public Health 7:242. https://doi.org/10.3389/fpubh.2019.00242

16. Hershberg R (2015) Mutation-The Engine of Evolution: Studying Mutation and Its Role in the Evolution of Bacteria: Figure 1. Cold Spring Harb Perspect Biol 7:a018077. https://doi.org/10.1101/cshperspect.a018077

17. Huang DW, Sherman BT, Lempicki RA (2009) Systematic and integrative analysis of large gene lists using DAVID bioinformatics resources. Nat Protoc 4:44-57. https://doi.org/10.1038/nprot.2008.211

18. Hudson RE, Bergthorsson U, Roth JR, Ochman H (2002) Effect of Chromosome Location on Bacterial Mutation Rates. Mol Biol Evol 19:85-92. https://doi.org/10.1093/oxfordjournals.molbev.a003986

19. Jahn LJ, Munck C, Ellabaan MMH, Sommer MOA (2017) Adaptive Laboratory Evolution of Antibiotic Resistance Using Different Selection Regimes Lead to Similar Phenotypes and Genotypes. Front Microbiol 8:816. https://doi.org/10.3389/fmicb.2017.00816

20. Jedrey H, Lilley KS, Welch M (2018) Ciprofloxacin binding to GyrA causes global changes in the proteome of Pseudomonas aeruginosa. FEMS Microbiol Lett 365:. https://doi.org/10.1093/femsle/fny134

21. Jiang C, Zhao Z (2006) Mutational spectrum in the recent human genome inferred by single nucleotide polymorphisms. Genomics 88:527-534. https://doi.org/10.1016/j.ygeno.2006.06.003

22. Jørgensen KM, Wassermann T, Jensen $P \emptyset$, et al (2013) Sublethal Ciprofloxacin Treatment Leads to Rapid Development of High-Level Ciprofloxacin Resistance during Long-Term Experimental Evolution of Pseudomonas aeruginosa. Antimicrob Agents Chemother 57:4215-4221. https://doi.org/10.1128/AAC.00493-13

23. Kim M, Weigand MR, Oh S, et al (2018) Widely Used Benzalkonium Chloride Disinfectants Can Promote Antibiotic Resistance. Appl Environ Microbiol 84:e01201-18, /aem/84/17/e01201-18.atom. https://doi.org/10.1128/AEM.01201-18

24. Kim S, Lieberman TD, Kishony R (2014) Alternating antibiotic treatments constrain evolutionary paths to multidrug resistance. Proc Natl Acad Sci 111:14494-14499. https://doi.org/10.1073/pnas.1409800111

25. Kirby WMM (1945) BACTERIOSTATIC AND LYTIC ACTIONS OF PENICILLIN ON SENSITIVE AND RESISTANT STAPHYLOCOCCI 1. J Clin Invest 24:165-169. https://doi.org/10.1172/JCl101593 
26. Krzywinski M, Schein J, Birol I, et al (2009) Circos: An information aesthetic for comparative genomics. Genome Res 19:1639-1645. https://doi.org/10.1101/gr.092759.109

27. Langmead B, Salzberg SL (2012) Fast gapped-read alignment with Bowtie 2. Nat Methods 9:357359. https://doi.org/10.1038/nmeth.1923

28. Lee L, Savage VM, Yeh PJ (2018) Intermediate Levels of Antibiotics May Increase Diversity of Colony Size Phenotype in Bacteria. Comput Struct Biotechnol J 16:307-315.

https://doi.org/10.1016/j.csbj.2018.08.004

29. Li B, Qiu Y, Shi H, Yin H (2016) The importance of lag time extension in determining bacterial resistance to antibiotics. The Analyst 141:3059-3067. https://doi.org/10.1039/C5AN02649K

30. Li H, Handsaker B, Wysoker A, et al (2009) The Sequence Alignment/Map format and SAMtools. Bioinformatics 25:2078-2079. https://doi.org/10.1093/bioinformatics/btp352

31. Lind PA, Berg OG, Andersson DI (2010) Mutational Robustness of Ribosomal Protein Genes. Science 330:825-827. https://doi.org/10.1126/science.1194617

32. Lyons DM, Lauring AS (2017) Evidence for the Selective Basis of Transition-to-Transversion Substitution Bias in Two RNA Viruses. Mol Biol Evol 34:3205-3215. https://doi.org/10.1093/molbev/msx251

33. Martincorena I, Seshasayee ASN, Luscombe NM (2012) Evidence of non-random mutation rates suggests an evolutionary risk management strategy. Nature 485:95-98. https://doi.org/10.1038/nature10995

34. Maxwell A, Bush NG, Evans-Roberts K (2015) DNA Topoisomerases. EcoSal Plus 6:. https://doi.org/10.1128/ecosalplus.ESP-0010-2014

35. Mi H, Muruganujan A, Ebert D, et al (2019) PANTHER version 14: more genomes, a new PANTHER GO-slim and improvements in enrichment analysis tools. Nucleic Acids Res 47:D419-D426. https://doi.org/10.1093/nar/gky1038

36. Miyata T, Miyazawa S, Yasunaga T (1979) Two types of amino acid substitutions in protein evolution. J Mol Evol 12:219-236. https://doi.org/10.1007/BF01732340

37. Moen B, Rudi K, Bore E, Langsrud S (2012) Subminimal Inhibitory Concentrations of the Disinfectant Benzalkonium Chloride Select for a Tolerant Subpopulation of Escherichia coli with Inheritable Characteristics. Int J Mol Sci 13:4101-4123. https://doi.org/10.3390/ijms13044101

38. Munck C, Gumpert HK, Wallin AIN, et al (2014) Prediction of resistance development against drug combinations by collateral responses to component drugs. Sci Transl Med 6:262ra156-262ra156. https://doi.org/10.1126/scitranslmed.3009940

39. Neher RA (2013) Genetic Draft, Selective Interference, and Population Genetics of Rapid Adaptation. Annu Rev Ecol Evol Syst 44:195-215. https://doi.org/10.1146/annurev-ecolsys-110512-135920

40. Oz T, Guvenek A, Yildiz S, et al (2014) Strength of Selection Pressure Is an Important Parameter Contributing to the Complexity of Antibiotic Resistance Evolution. Mol Biol Evol 31:2387-2401. https://doi.org/10.1093/molbev/msu191 
41. Pan F, Zhang H, Dong $X$, et al (2018) Comparative genomic analysis of multidrug-resistant Streptococcus pneumoniae isolates. Infect Drug Resist Volume 11:659-670. https://doi.org/10.2147/IDR.S147858

42. Payne JL, Menardo F, Trauner A, et al (2019) Transition bias influences the evolution of antibiotic resistance in Mycobacterium tuberculosis. PLOS Biol 17:e3000265. https://doi.org/10.1371/journal.pbio.3000265

43. Pietsch F, Bergman JM, Brandis G, et al (2017) Ciprofloxacin selects for RNA polymerase mutations with pleiotropic antibiotic resistance effects. J Antimicrob Chemother 72:75-84. https://doi.org/10.1093/jac/dkw364

44. Poole K (2012) Stress responses as determinants of antimicrobial resistance in Gram-negative bacteria. Trends Microbiol 20:227-234. https://doi.org/10.1016/j.tim.2012.02.004

45. Prasetyoputri A, Jarrad AM, Cooper MA, Blaskovich MAT (2019) The Eagle Effect and AntibioticInduced Persistence: Two Sides of the Same Coin? Trends Microbiol 27:339-354. https://doi.org/10.1016/j.tim.2018.10.007

46. Pu L, Jian Z, Pan F, et al (2019) Comparative genomic analysis and multi-drug resistance differences of Acinetobacter baumannii in Chongqing, China. Infect Drug Resist Volume 12:2827-2838. https://doi.org/10.2147/IDR.S216745

47. Ragupathi DNK, Veeraraghavan B, Sethuvel MDP, et al (2020) First Indian report on genome-wide comparison of multidrug-resistant Escherichia coli from blood stream infections. PLOS ONE 15:e0220428. https://doi.org/10.1371/journal.pone.0220428

48. Ramanathan B, Jindal HM, Le CF, et al (2017) Next generation sequencing reveals the antibiotic resistant variants in the genome of Pseudomonas aeruginosa. PLOS ONE 12:e0182524. https://doi.org/10.1371/journal.pone.0182524

49. Redgrave LS, Sutton SB, Webber MA, Piddock LJV (2014) Fluoroquinolone resistance: mechanisms, impact on bacteria, and role in evolutionary success. Trends Microbiol 22:438-445. https://doi.org/10.1016/j.tim.2014.04.007

50. Ricci V, Tzakas P, Buckley A, et al (2006) Ciprofloxacin-Resistant Salmonella enterica Serovar Typhimurium Strains Are Difficult To Select in the Absence of AcrB and TolC. Antimicrob Agents Chemother 50:38-42. https://doi.org/10.1128/AAC.50.1.38-42.2006

51. Roemhild R, Linkevicius M, Andersson DI (2020) Molecular mechanisms of collateral sensitivity to the antibiotic nitrofurantoin. PLOS Biol 18:e3000612. https://doi.org/10.1371/journal.pbio.3000612

52. Szklarczyk D, Gable AL, Lyon D, et al (2019) STRING v11: protein-protein association networks with increased coverage, supporting functional discovery in genome-wide experimental datasets. Nucleic Acids Res 47:D607-D613. https://doi.org/10.1093/nar/gky1131

53. Toprak E, Veres A, Michel J-B, et al (2011) Evolutionary paths to antibiotic resistance under dynamically sustained drug stress. Nat Genet 44:101-105. https://doi.org/10.1038/ng.1034

54. Vogel F, Kopun M (1977) Higher frequencies of transitions among point mutations. J Mol Evol 9:159-180. https://doi.org/10.1007/BF01732746 
55. Voumard M, Venturelli L, Borgatta M, et al (2020) Adaptation of Pseudomonas aeruginosa to constant sub-inhibitory concentrations of quaternary ammonium compounds. Environ Sci Water Res Technol 6:1139-1152. https://doi.org/10.1039/C9EW01056D

56. Wei Q, Tarighi S, Dötsch A, et al (2011) Phenotypic and Genome-Wide Analysis of an AntibioticResistant Small Colony Variant (SCV) of Pseudomonas aeruginosa. PLoS ONE 6:e29276. https://doi.org/10.1371/journal.pone.0029276

57. Wise R, Andrews JM, Edwards LJ (1983) In vitro activity of Bay 09867, a new quinoline derivative, compared with those of other antimicrobial agents. Antimicrob Agents Chemother 23:559-564. https://doi.org/10.1128/AAC.23.4.559

58. Zhang C-Z, Ren S-Q, Chang M-X, et al (2017) Resistance mechanisms and fitness of Salmonella Typhimurium and Salmonella Enteritidis mutants evolved under selection with ciprofloxacin in vitro. Sci Rep 7:9113. https://doi.org/10.1038/s41598-017-09151-y

59. Zhang J (2000) Rates of Conservative and Radical Nonsynonymous Nucleotide Substitutions in Mammalian Nuclear Genes. J Mol Evol 50:56-68. https://doi.org/10.1007/s002399910007

60. Zhang Z (2003) Patterns of nucleotide substitution, insertion and deletion in the human genome inferred from pseudogenes. Nucleic Acids Res 31:5338-5348. https://doi.org/10.1093/nar/gkg745

\section{Figures}

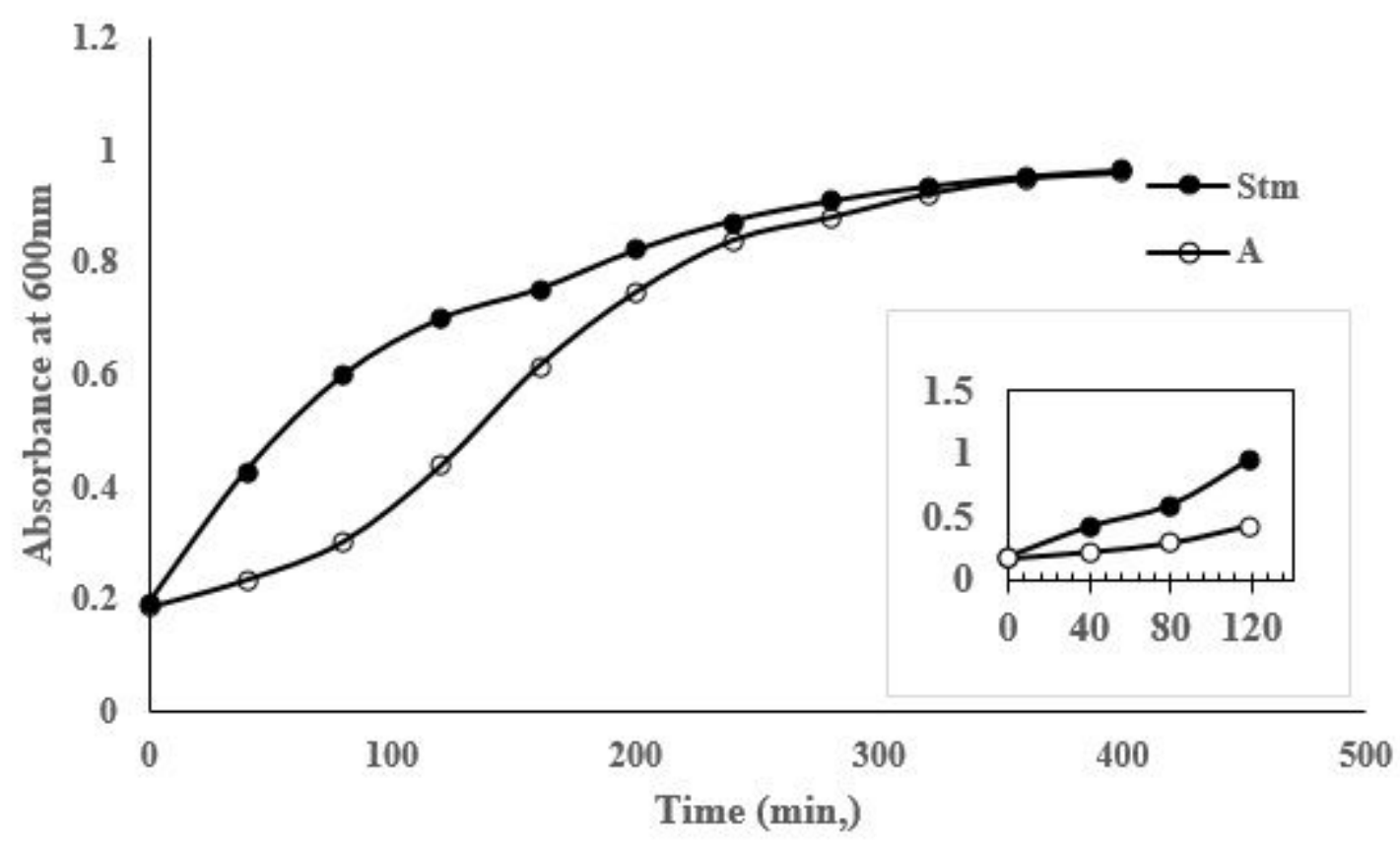

Figure 1 
Difference in the lag phases of wild type (Stm) and mutant (A) strains of S. Typhimurium. The lag phase was determined in LB medium. The growth analysis was carried out in 96-well microtiter plate at an absorbance of $600 \mathrm{~nm}$ on Synergy $\mathrm{H} 1$ microplate reader, Biotek (Winooski, Vermont, U.S.A). A medium shaking speed (120 rpm) was used and optical densities recorded after every 20 min for $20 \mathrm{hrs}$. at $37^{\circ} \mathrm{C}$. The $Y$ axis shows absorbance value at $600 \mathrm{~nm}$ and $X$ axis represents time interval in minutes (min.) The Clone A strain shows a lag of 70 min compared to the wild type S. Typhimurium (Stm) strain. The inset represents plot of $120 \mathrm{~min}$ where actual lag phase is clearly visible

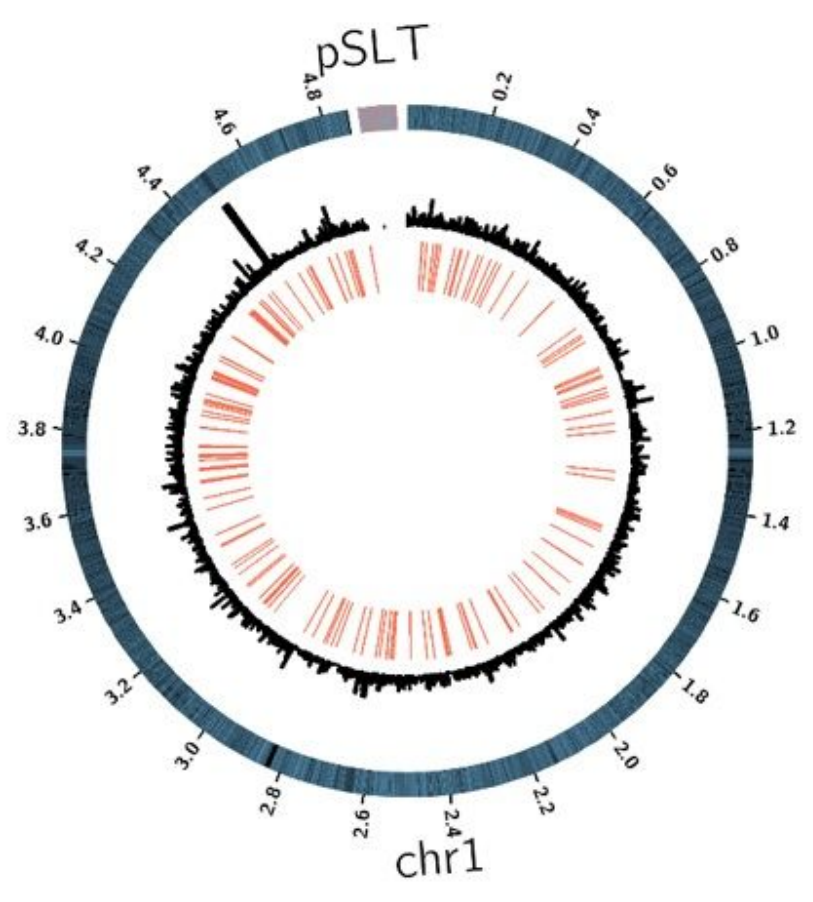

(A)

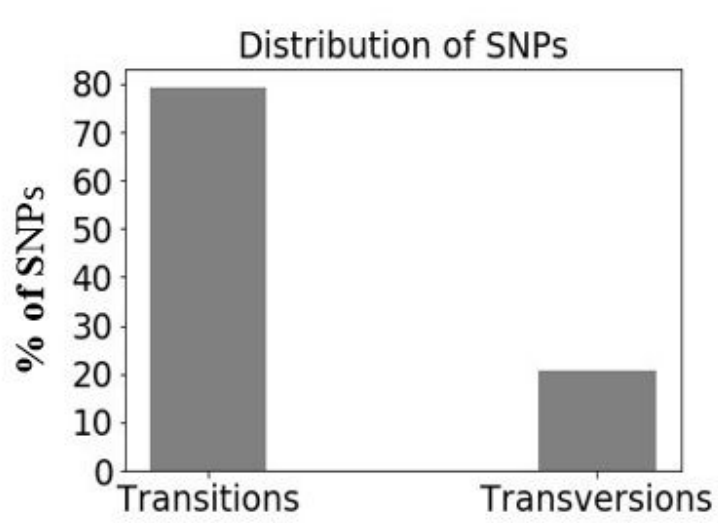

(B)

\section{Figure 2}

Overall distribution of mutations. (A) the CIRCOS plot shows the distribution of the mutations including SNPs and indels. The Salmonella genome consists of a chromosome (chr1) and a plasmid (pSLT1). The outermost ideogram shows the map of the genome with the genes marked in black stripes. The middle ideogram shows distribution of the SNPs as a histogram plot in black. The innermost ideogram shows distribution of the insertions and deletions in red. (B) Bar plot shows the percentage of transitions and transversions among the SNPS 


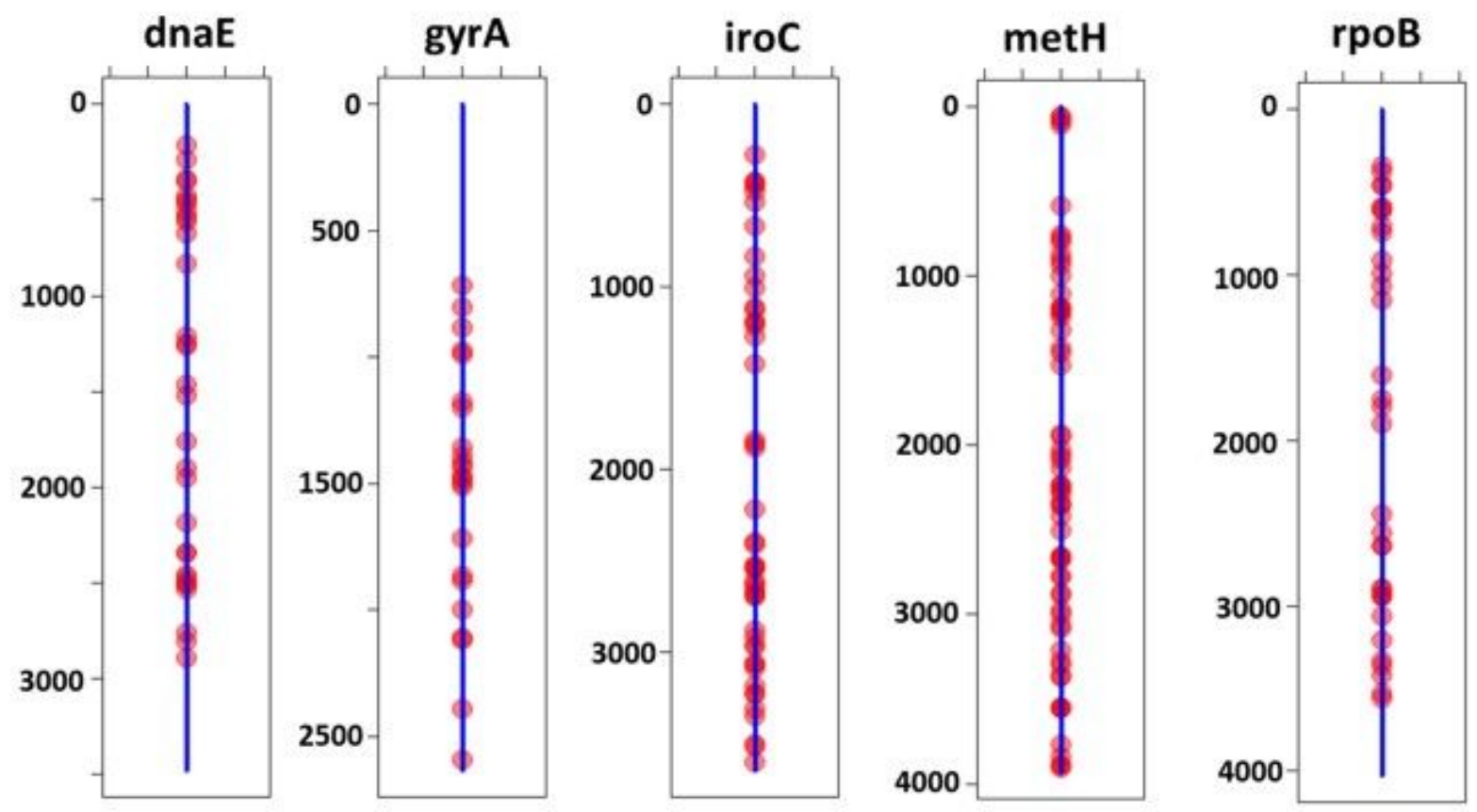

\section{Figure 3}

The distribution of mutations on individual genes are shown. The numbers indicate the position of the nucleotide. Each mutation is mapped as a red circle, and the gene is depicted as a blue line. These genes were among the highly mutated outlier population of all the mutated genes and also are important for mutator phenotype or antibiotic resistance. dnaE - DNA polymerase III, gyrA - DNA gyrase, iroC- Iron transporter, metH- methionine synthase, rpoB- RNA polymerase $B$ 

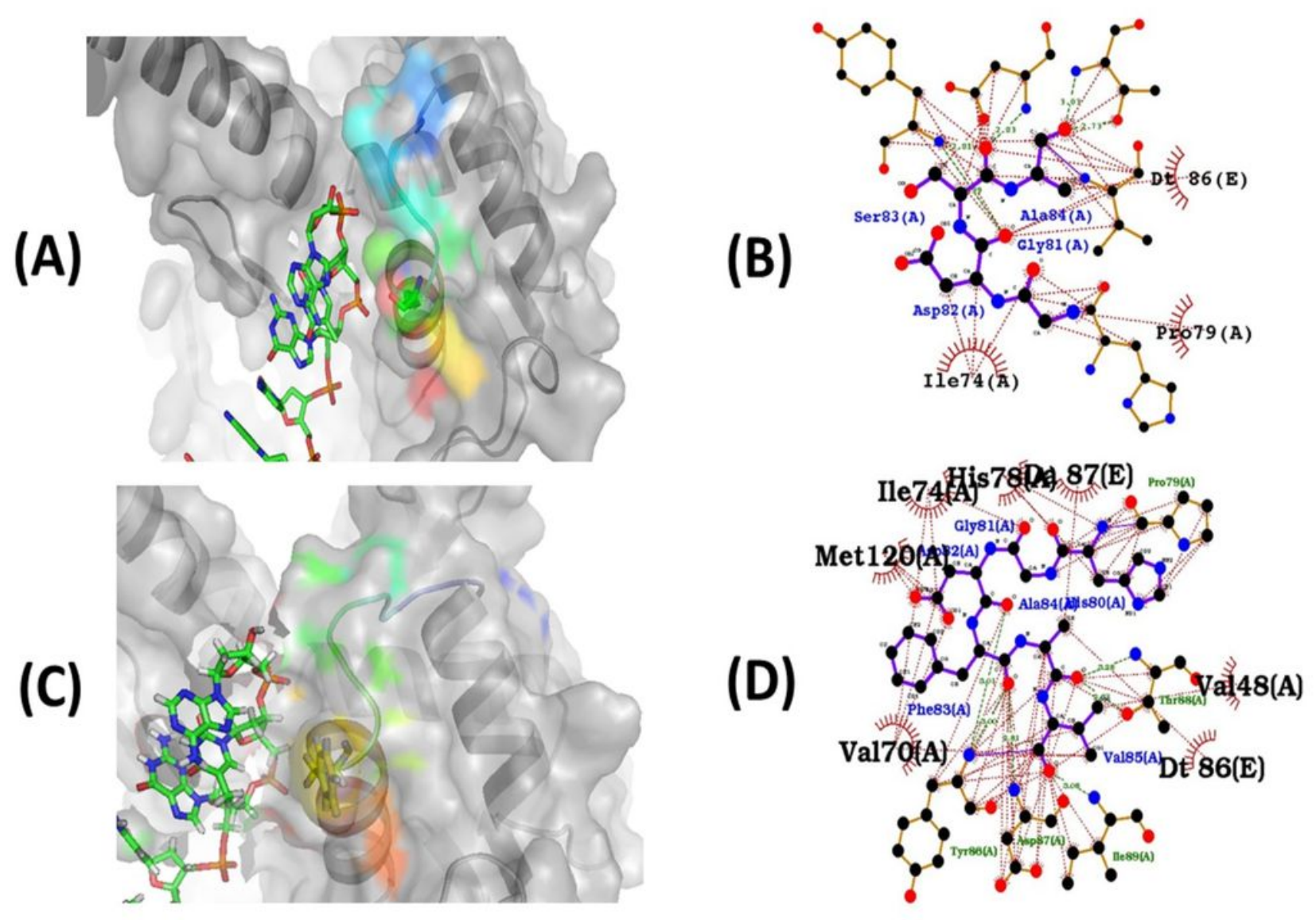

Figure 4

Homology model of gyrA was built by intensive modelling at PHYRE2 server. (A) wt gyrA with ser83. ala84 is in contact with the DNA and residues have limited hydrogen bonding (B) as seen in the LIGPLOT drawing. (C) the bulky phe83 replaces ser83. the hydrogen bonds in the vicinity of the residue (D) as seen in the LIGPLOT drawing has increased significantly creating a narrow and rigid pocket for the DNA 


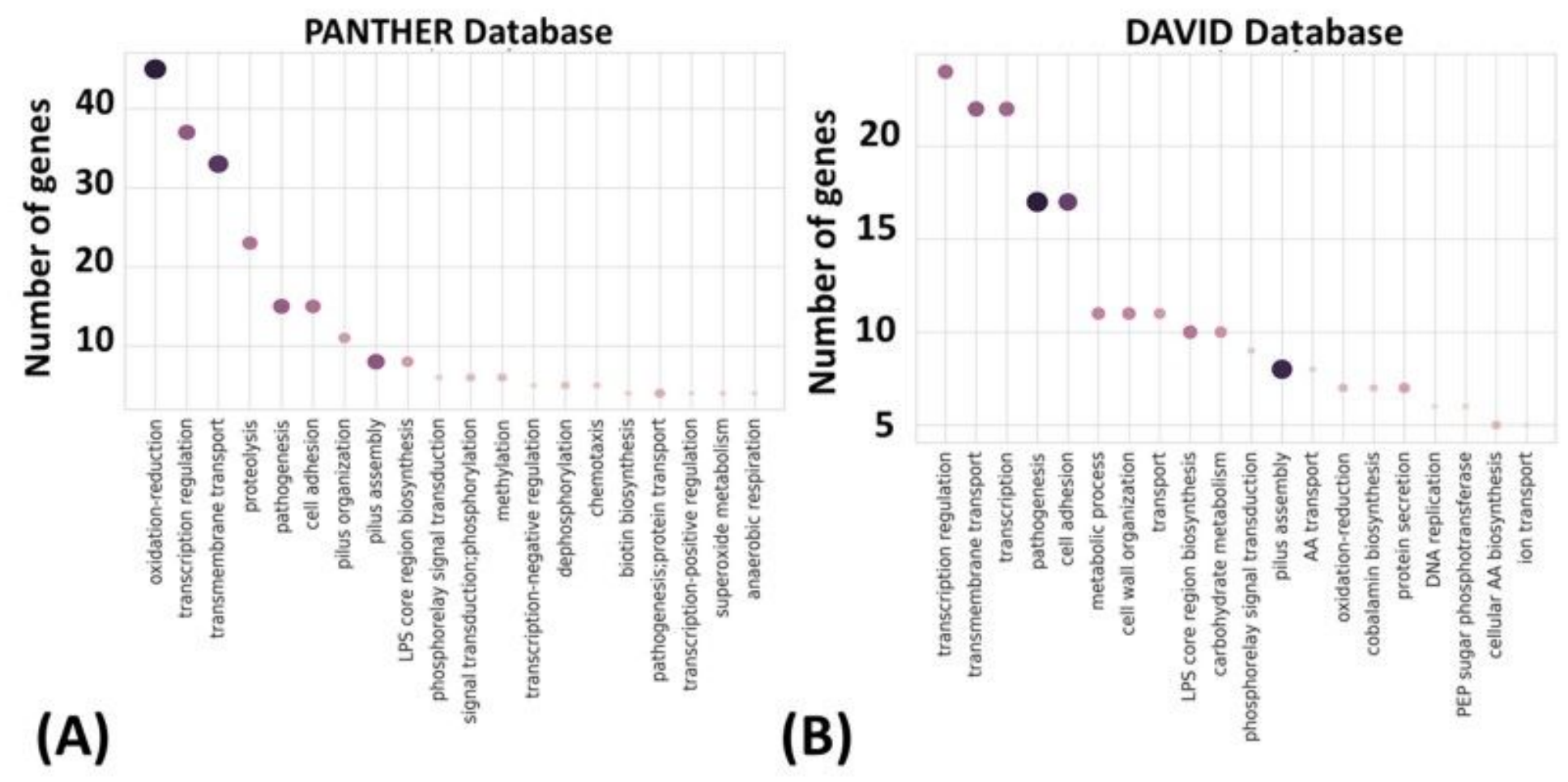

Figure 5

The genes with non-synonymous mutations were classified into pathways to find the pathways critical for ciprofloxacin resistance. The size and shade of the markers indicate the sum total of mutations in the genes of the pathway. A smaller size and lighter shade indicate fewer mutations while a darker shade and bigger size indicates more mutations (A) The mutated genes were classified using data from the Panther Database 9.0 at pantherdb.org. (B) The mutated genes were classified using data from the DAVID 6.8 database at david.ncifcrf.orf

\section{Supplementary Files}

This is a list of supplementary files associated with this preprint. Click to download.

- Fig.S1.docx

- Fig.S2.docx

- SupplementatyMM.docx

- TableS1.docx

- TableS2.xIsx 